\title{
Deep-vein thrombosis in the era of high HIV and tuberculosis prevalence: A prospective review of its diagnosis and treatment in a quaternary centre
}

\author{
K E Hodkinson, MB BCh; J N Mahlangu, MB BCh, FCPath (SA), MMed (Haem), Cert Clin Haem (SA) \\ Department of Molecular Medicine and Haematology, Faculty of Health Sciences, University of the Witwatersrand, Johannesburg; \\ and National Health Laboratory Service, Johannesburg, South Africa
}

Corresponding author: KE Hodkinson (drkatehodkinson@gmail.com)

\begin{abstract}
Background. Venous thromboembolic disease (VTE) is a leading cause of morbidity and mortality worldwide. HIV and tuberculosis (TB) infections have an aetiological association with VTE. Implementation of national HIV and TB programmes in South Africa (SA) has changed the burden of these two conditions, with resultant effects on VTE prevalence. Furthermore, with the increased use of direct oral anticoagulants (DOACs), baseline thrombosis data are needed to evaluate the effect of these new agents.

Objectives. To determine real-life baseline VTE characteristics in a pre-DOAC era, and to document the association of HIV and TB infections with VTE.

Methods. This was a single-centre prospective cohort study performed in a quaternary care centre at Charlotte Maxeke Johannesburg Academic Hospital, SA. Key inclusion criteria included signed informed consent by adults ( $\geq 18$ years) with a new episode of thrombosis. Procedures included physical examination, thrombosis risk factor assessment, duplex Doppler examination, thrombophilia screening, inpatient treatment and outpatient follow-up.

Results. Ninety-nine participants with confirmed thrombosis met the inclusion criteria. Participants were predominantly black (79.8\%) and female (64.6\%), with a median age of 46 (interquartile range (IQR) 38 - 57) years. The prevalences of HIV and TB were $53.0 \%$ and $21.2 \%$, respectively. The most common risk factors for thrombosis were TB (17.2\%) and malignancies (14.1\%). Thrombophilia screening had a low diagnostic yield. The median time to target international normalised ratio during hospitalisation was 5.5 (IQR 4.0 - 7.0) days and the median duration of hospitalisation was 9 (IQR 7 - 11) days. The overall mortality rate in the cohort at 3 months post hospitalisation was $12.1 \%$. Conclusions. This prospective study provides real-life data on thrombosis diagnosis and management at a quaternary public healthcare facility, providing a valuable baseline against which the effect of new DOAC anticoagulants can be assessed. Further research is required to clarify the aetiological association between thrombosis and HIV and TB.
\end{abstract}

S Afr Med J 2017;107(10):859-863. DOI:10.7196/SAMJ.2017.v107i10.12443

Venous thromboembolic disease (VTE), comprising deep-vein thrombosis (DVT) and pulmonary embolism, is a leading cause of morbidity and mortality worldwide. ${ }^{[1-2]}$ The prevalence of VTE in the South African (SA) population is currently poorly characterised and largely unknown.

In SA, there is a high dual burden of HIV and tuberculosis (TB), both of which have well-established associations with the development of VTE. There are several plausible explanations for the prothrombotic effect of these infections. These include induction of tumour necrosis factor alpha and interleukin-6 production by chronic infections, which renders the vascular endothelium thrombogenic, interference with the normal production of coagulation factors in the liver, an increase in the levels of factor VIII, fibrinogen and plasminogen activator inhibitor-1, and a reduction in antithrombin and protein C. ${ }^{[3-5]}$ The causal relationship between TB and VTE is demonstrated in part by an improvement in the hypercoagulable state within 1 month of initiating anti-TB therapy. ${ }^{[4]}$

Recently implemented national diagnostic and treatment programmes in SA are rapidly changing the landscape of both HIV and $\mathrm{TB}^{[6-7]}$ Changes include an improvement in TB cure rates to $\sim 80 \%$, a $\sim 20 \%$ reduction in the number of new HIV cases, and an increase in antiretroviral therapy (ART) coverage. ${ }^{[6]}$

It is intended to introduce direct oral anticoagulants (DOACs) for the treatment of DVT in quaternary healthcare institutions. Their advantages include more predictable pharmacokinetics, allowing for standardised dosing and less frequent follow-up, which may improve long-term anticoagulation and compliance rates. ${ }^{[8]}$ The current treatment of VTE with warfarin anticoagulation is challenging in the setting of comorbid HIV and/or TB because of the many drug interactions, particularly in patients on treatment for $\mathrm{TB}$, in whom therapeutic international normalised ratio (INR) values can be difficult to achieve ${ }^{[9]}$ With the introduction of new DOACs it will be important to obtain baseline data on VTE prevalence, diagnosis and treatment in order to evaluate the benefit of these anticoagulants in an era of high HIV and TB prevalence and incidence.

\section{Objectives}

To characterise the clinical presentation, diagnosis, treatment and follow-up of VTE, with specific consideration of its association with HIV and TB infections, in a quaternary public sector healthcare setting in SA.

\section{Methods}

\section{Study design and study population}

This was a single-centre, prospective cohort study performed at Charlotte Maxeke Johannesburg Academic Hospital (CMJAH), a quaternary healthcare facility. The study, conducted from November 2015 to January 2017, was reviewed and approved by the University 
of the Witwatersrand Human Research Ethics Committee (ref. no. M150938) as well as the CMJAH management.

Study participants were unselected patients presenting to the Haematology Unit at CMJAH. Key inclusion criteria included signed informed consent by adults ( $\geq 18$ years) with a new episode of DVT. These patients came to the Haematology Unit via the CMJAH Emergency Unit or through referrals from other centres, or were inpatients for whom the Haematology Unit was consulted to manage the DVT.

Physical examination and thrombosis pretest probability assessments (Wells score) were performed, and thrombosis was confirmed using a duplex Doppler examination (DDE). HIV status was established according to institutional procedure. Where indicated, thrombophilia screening was performed as part of the routine diagnostic work-up. Data collected included past medical history, concurrent diseases and treatment history, and blood samples were taken for routine haematology, coagulation and chemistry tests as per standard of care. These data were captured on a data collection sheet and anonymised prior to analysis.

\section{Statistical analysis}

Statistical analysis was performed using Stata 14 (StataCorp, USA) with the help of a statistician. Quantitative data were summarised in tables and figures and described using medians with interquartile ranges (IQRs), counts with percentages, Fisher's exact test and the Mann-Whitney $U$-test. Statistical significance was set at $p<0.05$.

\section{Results}

\section{Demographics and clinical presentation}

During the 15-month study period, 126 individuals were referred to the Haematology Unit at CMJAH with clinical symptoms of DVT. Thrombosis was confirmed in 99 of these, and their pertinent demographic data are summarised in Table 1.

The clinical presentation was both pain and swelling of the limb in 60 participants $(60.1 \%)$, with the remainder reporting either limb pain $(n=5)$ or swelling $(n=34)$. None of the participants were admitted specifically for symptoms of HIV or TB.

\section{Diagnostic work-up}

The thrombosis risk profile of the study population, stratified by HIV and TB status, is shown in Table 2 .

The median Wells score was 3 (IQR 2 - 4). In the majority of cases the thrombosis occurred in the lower limb $(n=97)$, with only two participants having upper limb thromboses.

The median D-dimer value in 93 participants tested was 1.71 (1 3.67) $\mathrm{mg} / \mathrm{L}$. Four participants had a negative D-dimer result despite demonstrable thrombosis on DDE. The median waiting period for a DDE in 88 evaluable participants was 30 (24 - 36) hours, and 20 participants waited $>48$ hours. DDE of 11 participants was done by the referring hospital or at CMJAH during admission, for another reason, prior to admission to the Haematology Unit.

Assays to investigate the cause of the thrombosis were performed in 64 participants on admission. All 64 had clotting assays done, while only 40 had molecular tests performed. The thrombophilia screening results are summarised in Table 3 . The 64 clotting assays performed revealed a total of six abnormal results. There were no abnormal molecular results.

\section{Management of thrombosis}

Table 4 shows the follow-up and outcomes of the study participants. Of the 99 participants, 91 were initiated on a low-molecular-weight
Table 1. Demographic data and baseline characteristics of participants $(N=99)$

\begin{tabular}{|c|c|}
\hline Age (years), median (IQR) & $46(38-57)$ \\
\hline \multicolumn{2}{|l|}{ Sex, $n(\%)$} \\
\hline Male & $35(35.4)$ \\
\hline Female & $64(64.6)$ \\
\hline \multicolumn{2}{|l|}{ Race, $^{*} n(\%)$} \\
\hline Black & $79(79.8)$ \\
\hline White & $13(13.1)$ \\
\hline Coloured & $4(4.0)$ \\
\hline Indian & $3(3.0)$ \\
\hline \multicolumn{2}{|l|}{ Thrombosis investigation, $n$ (\%) } \\
\hline Duplex doppler confirmation & $99(100)$ \\
\hline СТPA & $5(5.1)$ \\
\hline Ventilation/perfusion scan & $1(1.0)$ \\
\hline \multicolumn{2}{|l|}{$\mathrm{HIV}^{\dagger}$ diagnosis and treatment, $n(\%)$} \\
\hline Positive & $44 / 83(53.0)$ \\
\hline Negative & $39 / 83(47.0)$ \\
\hline On treatment & $34 / 44(77.3)$ \\
\hline Not on treatment ${ }^{\ddagger}$ & $10 / 44(22.7)$ \\
\hline $\mathrm{TB}^{\varsigma}$ diagnosis and treatment, $n(\%)$ & $21(21.2)$ \\
\hline On active treatment & $17 / 21(81.0)$ \\
\hline Not on treatment & $4 / 21(19.0)$ \\
\hline
\end{tabular}

anticoagulant, enoxaparin sodium, and then given both enoxaparin sodium and a vitamin $\mathrm{K}$ antagonist, warfarin, once the diagnosis of thrombosis was made. The starting dose of warfarin was $5 \mathrm{mg}$ with no loading dose. Enoxaparin sodium was given subcutaneously at $1 \mathrm{mg} / \mathrm{kg}$ twice daily after admission. Once thrombosis was confirmed, enoxaparin sodium was continued for at least 5 days following the initiation of concomitant warfarin anticoagulation. Five participants were on enoxaparin sodium alone without warfarin - these were patients with malignancy $(n=3)$ and those who were pregnant $(n=2)$. Warfarin was stopped as soon as the INR was in the therapeutic range 2 - 3. Enoxaparin sodium was discontinued after a minimum of 5 days and once a therapeutic INR had been achieved. The median warfarin dose per week required to obtain a therapeutic INR was 30 (IQR 20 - 43) $\mathrm{mg}$ and the median time to a therapeutic INR was 5.5 (IQR 4.0 - 7.0) days. Of the 14 participants with cancer-associated thrombosis, only three (21.4\%) were treated with enoxaparin sodium, which is the preferred treatment modality. ${ }^{[10-13]}$ The median duration of hospitalisation in the cohort was 9 (IQR 7 - 11) days.

All participants were referred to the CMJAH INR clinic upon discharge for long-term follow-up. Of the 91 participants on warfarin, 79 returned for follow-up at 1 month. During this period, adequate duration and consistency of anticoagulation therapy was achieved by 57 participants $(72.2 \%)$. Eleven participants were lost to followup. Twelve of the 99 participants (12.1\%) died during the study period.

\section{Comorbid HIV and TB infections}

HIV status was established in 83 of the 99 participants. Fortyfour (53.0\%) were HIV-positive, with a median CD4 count of 152 (IQR 85 - 493) cells $/ \mu \mathrm{L}$ and a median viral load of 482 (IQR 23 221250 ) copies/mL. Thirty-four of the $44 \mathrm{HIV}$-positive participants (77.3\%) were on ART, three starting treatment while in hospital after the diagnosis of their DVT. Of the 10 participants who were not 


\section{Table 2. Risk factors for DVT by HIV and TB infection $(N=99)$}

\begin{tabular}{|c|c|c|c|c|}
\hline & $\begin{array}{l}\text { HIV+ } \\
(N=24), n\end{array}$ & $\begin{array}{l}\text { HIV+ and TB+ } \\
(N=20), n\end{array}$ & $\begin{array}{l}\text { HIV- } \\
(N=39), n\end{array}$ & $\begin{array}{l}\text { HIV unknown } \\
(N=16), n\end{array}$ \\
\hline Major general surgery & 0 & 0 & 3 & 2 \\
\hline Orthopaedic surgery & 0 & 0 & 0 & 1 \\
\hline Hospitalised for $\geq 3$ days & 1 & 0 & 1 & 0 \\
\hline Malignancy & 5 & 0 & 7 & 2 \\
\hline Paralysis/stroke & 2 & 0 & 1 & 2 \\
\hline TB treatment & 0 & 16 & 1 & 0 \\
\hline Lower-limb cast & 0 & 0 & 0 & 2 \\
\hline Oral contraceptive & 0 & 2 & 5 & 0 \\
\hline Pregnancy & 1 & 0 & 1 & 0 \\
\hline Postpartum & 0 & 0 & 2 & 0 \\
\hline Central line & 0 & 1 & 2 & 0 \\
\hline Long journey of 4 - 6 hours & 0 & 0 & 1 & 3 \\
\hline
\end{tabular}

Table 3. Assays for investigation of thrombosis aetiology

\begin{tabular}{ll}
\hline Thrombosis assay & $\begin{array}{l}\text { Assays with abnormal } \\
\text { results, } \boldsymbol{n}\end{array}$ \\
\hline Clotting assays $(N=64)$ & \\
Protein $C$ & 5 \\
Protein $S$ & 4 \\
Antithrombin & 2 \\
APCR & 0 \\
Molecular assays $(N=40)$ & \\
Factor V Leiden & 0 \\
Prothrombin gene $(G 2021 A)$ & 0 \\
APCR = activated protein C resistance. &
\end{tabular}

\section{Table 4. Patient follow-up during the study}

\begin{tabular}{ll}
\hline Phases of follow-up & Patients, $\boldsymbol{n}$ \\
\hline Screening phase & \\
$\quad$ Screened for thrombosis & 126 \\
Confirmed thrombosis & 99 \\
Deaths & 0 \\
In-hospital follow-up & \\
$\quad$ Treated with VKA + LMWH & 91 \\
Treated with LMWH only & 5 \\
Deaths* & 3 \\
Post-hospitalisation follow-up & 19 \\
$\quad<3$ months & 57 \\
$\geq 3$ months & 11 \\
Lost to follow-up & 9 \\
Deaths & \\
VKA = vitamin K antagonist; LMWH = low-molecular-weight heparin. \\
${ }^{*}$ Probable causes of death were malignancy $(n=8)$, sepsis $(n=3)$ and stroke $(n=1)$.
\end{tabular}

initiated on ART in hospital, two were not eligible, as they had just started TB treatment.

Of the 99 participants, $21(21.2 \%)$ had a diagnosis of TB infection; in four cases this was diagnosed during hospitalisation. Seventeen participants were receiving $\mathrm{TB}$ treatment at the time of DVT diagnosis. HIV and TB co-infection was present in 20 participants. This group had a lower median CD4 count (118 (IQR 60 - 191) cells/ $\mu \mathrm{L}$ ) than their HIV-positive, TB-negative counterparts (321 (IQR 92 - 545) cells $/ \mu \mathrm{L})(p<0.05)$.

\section{Discussion}

This prospective cohort study provided baseline data on the clinical presentation, diagnostic work-up and management of 99 patients with DVT with or without HIV and/or TB infections in a quaternary public healthcare facility.

The median age of the patient cohort was 46 years, which reflects the age distribution in the adult haematology practice where the study was performed. This age is higher than the age of peak HIV prevalence at CMJAH, which is in the second and third decades. The racial distribution of the cohort mirrors that of the users of public healthcare facilities in SA. These figures would be different in the private healthcare sector and therefore do not reflect the true prevalence of thrombosis in the SA general population.

The clinical presentation of thrombosis in our patient cohort is similar to that elsewhere in the world. ${ }^{[14]}$ Fifty-three percent of participants with thrombosis were also HIV-positive. This figure is similar to HIV-positivity rates in many public healthcare facilities in SA. ${ }^{[15-16]}$

TB was the most common risk factor for thrombosis in participants who were also HIV-positive. The burden of HIV and TB has begun to change in SA with the implementation of national diagnostic and treatment programmes. It remains to be seen whether this will in turn have an impact on the prevalence of thrombosis.

The work-up of participants with suspected DVT included the use of a clinical scoring system (the Wells score), and a quantitative D-dimer test, with confirmation on DDE. Studies have shown that DVT can safely be excluded in patients with the combination of a low Wells score and a negative quantitative D-dimer. ${ }^{[17-19]}$ In our cohort, all four participants with a negative D-dimer had an intermediate Wells score and therefore went on to have a DDE to confirm thrombosis. The value of routinely using such an approach would be to limit the number of unnecessary DDEs.

Thrombophilia screening was performed in 64 participants, and six results were abnormal. This result is low relative to published studies, but the numbers are too small to establish an explanation. ${ }^{[20]}$ Molecular tests for factor V Leiden and prothrombin G20210A mutations were negative in all 35 black participants in our study, a finding in keeping with previous studies confirming the absence of the factor V Leiden mutation in this population. ${ }^{[21-23]}$

Warfarin and enoxaparin sodium are currently the most widely used anticoagulants for the treatment of DVT in SA public hospitals. Their use at CMJAH was comparable to published national and international guidelines. ${ }^{[24-28]}$ Enoxaparin sodium discontinuation 
procedures were adhered to in only $38 \%$ of participants. This low adherence is explained by both hospital- and patient-related factors, which included chronic bed shortages at CMJAH and inability of participants to be absent from work for extended periods. A more achievable target was therefore to obtain a minimum of one therapeutic INR and to administer at least 5 days of enoxaparin sodium before discontinuation. The use of enoxaparin sodium in cancer-associated thrombosis was low $(21 \%)$ in our cohort. Plausible explanations include cost, lack of awareness of the current guidelines, clinician habit, safety concerns and the burden of daily injections for cancer patients. ${ }^{[29]}$

In the current study, the average duration of hospitalisation required to achieve a therapeutic INR was 1 - 2 weeks. Adequate and consistent long-term anticoagulation therapy was achieved in 57 (72.2\%) of the cohort, as shown in Table 4. Possible reasons for inadequate and inconsistent anticoagulation in the remaining $30 \%$ may include participants being unable to take time off work for follow-up visits and unaffordable transport costs. ${ }^{[30]}$

The introduction of DOAC therapy has the potential to reduce or eliminate the need for hospitalisation and testing, thus providing substantial cost savings for both hospitals and patients. However, studies predict that there will be significant drug interactions between DOACs and certain HIV and TB medications. There are known interactions with strong CYP3A4 inducers and inhibitors, namely rifampicin (standard of care) and protease inhibitors. ${ }^{[31-33]}$ Based on these predictions, anticoagulation of patients with TB is likely to remain a challenge, as is currently experienced with warfarin anticoagulation. It is therefore anticipated that the benefits of DOACs may not be fully realised in patients with $\mathrm{TB}$, and they are yet to be determined in those with HIV.

The HIV prevalence rate of $53.0 \%$ in our cohort was notably higher than the sub-Saharan African prevalence of $19.2 \% .{ }^{[34]}$ This finding probably reflects the prothrombotic risk associated with HIV. In addition to the direct effect of HIV on DVT development, opportunistic infections and their treatments, with associated prolonged bed rest and malignancies, are possible contributing factors in this subgroup. A significant proportion of the participants were on ART at the time of DVT diagnosis (77.3\%), which indicates the efficiency of the national antiretroviral roll-out programme in SA. ART has been shown to reduce markers of coagulation, but abnormalities persist despite viral suppression. Twenty-one participants had comorbid HIV and TB, which reflects the scenario in the general population in this region. As expected, CD4 counts in this group were significantly lower than those in their TB-negative counterparts. Satisfactory anticoagulation therapy is a huge challenge in patients with $\mathrm{TB}$ infection owing to the unpredictable effects of rifampicin and isoniazid on the cytochrome P450 system, which is involved in warfarin metabolism.

Twelve participants (12.1\%) died at various time points during the study, as shown in Table 4 . The majority of the deaths occurred during post-hospitalisation follow-up. The probable causes of death in these participants were malignancies $(n=8)$, sepsis $(n=3)$ and recent stroke $(n=1)$. The malignancies were signet ring cell adenocarcinoma, mesothelioma, breast, cervical, prostatic and bronchoalveolar carcinoma, and an unknown primary tumour. While no deaths due to thrombosis or bleeding complications were documented in the study, these remain possibilities for the 11 participants who were lost to follow-up.

\section{Study limitations}

The small patient population of 99 is an obvious limitation of this study. Our study figures therefore cannot be extrapolated to the general hospital population and may not reflect thrombosis as a whole in a quaternary care setting. The majority of the participants in this study had lower-limb thromboses. We would not have seen patients with other sites of thrombosis, such as intra-abdominal and pulmonary, if they were managed by other disciplines of medicine. Finally, the follow-up of 3 months was short and may not reflect the true outcome of management of thrombosis in a quaternary care setting in the long term.

\section{Conclusion}

This prospective study provides real-life data on the prevalence of DVT in the pre-DOAC era. The data will serve as a baseline reference for measuring the effectiveness and impact of the new DOACs when used routinely outside clinical trials. This is of particular interest in patients with comorbid HIV and TB, where conventional anticoagulation treatment is challenging. The prevalences of HIV and TB observed in this study provide an important reference point for assessing the aetiological association between thrombosis and these infections, especially as their prevalence changes as a result of the current national interventions. To elucidate the aetiological association of HIV and TB with thrombosis fully, further research into the underlying pathophysiological mechanisms is required.

Acknowledgements. We thank Prof. Piet Becker for the statistical analysis, Mr Wayne K Glossop for assistance with editing, and Sister Johannah Sithole, Dr Jenique Bailey and Dr Esihle Nomlomo for assistance with data collection.

Author contributions. KH conceived the study topic, wrote the study protocol, collected data, analysed the data, wrote the article and approved the final version. JM approved the study conception, supervised the study conduct and data collection, analysed the data, wrote the article and approved the final version.

Funding. None.

Conflicts of interest. None.

1. ISTH Steering Committee for World Thrombosis Day. Thrombosis: A major contributor to the global disease burden. J Thromb Haemost 2014;12(10):1580-1590. https://doi.org/10.1111\%2Ftth.12698

Beckman MG, Hooper WC, Critchley SE, Ortel TL. Venous thromboembolism: A public health concern. Am J Prev Med 2010;38(4):S495-S501. https://doi.org/10.1016\%2Fj.amepre.2009.12.017

3. Robson SC, White NW, Aronson I, Woollgar R, Goodman H, Jacobs P. Acute-phase response and . Robson SC, White NW, Aronson I, Woollgar R, Goodman H, Jacobs P. Acute-phase response and
the hypercoagulable state in pulmonary tuberculosis. Br J Haematol 1996;93(4):943-949. https://doi. the hypercoagulable state in pulmonary tube
org $10.1046 \% 2 \mathrm{Fj} .1365-2141.1996 . \mathrm{d} 01-1722 . \mathrm{x}$

4. Turken O, Kunter E, Sezer M, et al. Hemostatic changes in active pulmonary tuberculosis. Int $\mathrm{J}$ Tuberc Lung Dis 2002;6(10):927-932.

5. Louw S, Jacobson BF, Büller H. Human immunodeficiency virus infection and acute deep vein thromboses. Clin Appl Thromb Hemostat 2008;14(3):352-355. http://dx.doi. rg/ $/ 10.1177 / 1076029607304411$

6. South African National AIDS Council. National Strategic Plan on HIV, TB and STIs 2017 - 2022. Pretoria: SANAC, 2017. http://sanac.org.za/wp-content/uploads/2017/05/NSP_FullDocument_ FINAL.pdf (accessed 18 April 2017).

7. Stevens WS, Scott L, Noble L, Gous N, Dheda K. Impact of the GeneXpert MTB/RIF technology on tuberculosis control. Microbiol Spectr 2017;5(1). https://doi.org/10.1128\%2Fmicrobiolspec.tbtb20040-2016

8. Yeh CH, Gross PL, Weitz JI. Evolving use of new oral anticoagulants for treatment of venous thromboembolism. Blood 2014;124(7):1020-1028. https:// doi.org/10.1182\%2Fblood-2014-03-563056 9. Lee CR, Thrasher KA. Difficulties in anticoagulation management during coadministration of warfarin and rifampin. Pharmacotherapy 2001;21(10):1240-1246. https://doi.org/10.1592\%2Fph warfarin and rifam
co.21.15.1240.33897

10. Hull RD, Pineo GF, Brant RF, et al. Long-term low-molecular-weight heparin versus usual care in proximal-vein thrombosis patients with cancer. Am J Med 2006;119(12):1062-1072. http://dx.doi.
. org/10.1016/j.jvs.2007.05.019

11. Deitcher SR, Kessler CM, Merli G, Rigas JR, Lyons RM, Fareed J. Secondary prevention of venous thromboembolic events in patients with active cancer: Enoxaparin alone versus initial enoxaparin followed by warfarin for a 180-day period. Clin Appl Thromb Hemostat 2006;12(4):389-396. http:// dx.doi.org/10.1177/1076029606293692

12. Lee AY, Levine MN, Baker RI, et al. Low-molecular-weight heparin versus a coumarin for the prevention of recurrent venous thromboembolism in patients with cancer. N Engl J Med 2003;349(2):146-153. http://dx.doi.org/10.1016/j.accreview.2003.08.061

13. Meyer G, Marjanovic Z, Valcke J, et al. Comparison of low-molecular-weight heparin and warfarin for the secondary prevention of venous thromboembolism in patients with cancer: A randomized controlled study. Arch Intern Med 2002;162(15):1729-1735. http://dx.doi.org/10.1001/archinte.162.15.1729

14. Kahn SR. The clinical diagnosis of deep venous thrombosis: Integrating incidence, risk factors, and symptoms and signs. Arch Intern Med 1998;158(21):2315-2323. https://doi.org/10.1001\%2Farchin te.158.21.2315 
15. Marchal B, de Brouwere V, Kegels G. Viewpoint: HIV/AIDS and the health workforce crisis: What are the next steps? Trop Med Int Health 2005;10(4):300-304. https://doi.org/10.1111\%2Fj.13653156.2005.01397.x

16. Awolesi D, Naidoo M, Cassimijee MH. The profile and frequency of known risk factors or comorbidities for deep vein thrombosis in an urban district hospital in KwaZulu-Natal. South Afr I HIV Med 2016;17(1). http://dx.doi.org/10.4102/hivmed.v17i1.425

17. Gaitini D, Khoury R, Israelit S, Beck-Razi N. Sparing ultrasound in emergency department patients with suspected deep vein thrombosis by using clinical scores and D-dimer testing. J Clin Ultrasound 2016;44(4):231-239. http://dx.doi.org/10.1002/jcu.22317

18. Rahiminejad M, Rastogi A, Prabhudesai S, et al. Evaluating the use of a negative D-dimer and modified low Wells score in excluding above knee deep venous thrombosis in an outpatient population, assessing need for diagnostic ultrasound. ISRN Radiol 2014(2014): article ID 519875. http://dx.doi. org/10.1155/2014/519875

19. Wells PS, Anderson DR, Rodger M, et al. Evaluation of D-dimer in the diagnosis of suspected deepvein thrombosis. N Engl J Med 2003;349(13):1227-1235. http://dx.doi.org/10.1056/nejmoa023153

20. Meyer MR, Witt DM, Delate T, et al. Thrombophilia testing patterns amongst patients with acute venous thromboembolism. Thromb Res 2015;136(6):1160-1164. http://dx.doi.org/10.1016/j. thromres.2015.10.019

21. Bauduer F, Lacombe D. Factor V Leiden, prothrombin 20210A, methylenetetrahydrofolate reductase 677T, and population genetics. Mol Genet Metab 2005;86(1-2):91-99. http://dx.doi.org/10.1016/j. ymgme.2005.04.002

22. Rubinstein R, Kolia F, Novitzky N. Prevalence of factor V Leiden in three ethnic groups of patients with deep vein thrombosis in the Western Cape province of South Africa. Eur J Haematol 2000;65(1):78-79. http://dx.doi.org/10.1034/j.1600-0609.2000.9c168.x

23. Rees DC, Cox M, Clegg JB. World distribution of factor V Leiden. Lancet 1995;346(8983):1133-1134. http://dx.doi.org/10.1016/s0140-6736(95)91803-5

24. Jacobson BF, Louw S, Büller H, et al. Venous thromboembolism: Prophylactic and therapeutic practice guideline. S Afr Med J 2013;103(4):261-267. http://dx.doi.org/10.7196/SAMJ.6706

25. Kearon C, Akl EA, Comerota AJ, et al. Antithrombotic therapy for VTE disease: Antithrombotic therapy and prevention of thrombosis, 9th ed: American College of Chest Physicians Evidence-Based Clinical Practice Guidelines. Chest 2012:141(2 Suppl):e419S-e494S. http//dx doi org/10.1378/chest 11-2301
26. Mandalà $\mathrm{M}$, Falanga $\mathrm{A}$, Piccioli $\mathrm{A}$, et al. Venous thromboembolism and cancer: Guidelines of the Italian Association of Medical Oncology (AIOM). Crit Rev Oncol Hematol 2006;59(3):194-204. http:// Italian Association of Medical Oncology (A.
dx.doi.org/10.1016/j.critrevonc.2006.05.001

27. Lyman GH, Khorana AA, Falanga A, et al. American Society of Clinical Oncology guideline Lyman GH, Khorana AA, Falanga A, et al. American Society of Clinical Oncology guideline:
Recommendations for venous thromboembolism prophylaxis and treatment in patients with cancer. Clin Oncol 2007;25(34):5490-5505. http://dx.doi.org/10.1200/jco.2007.14.1283

28. Farge D, Bosquet L, Kassab-Chahmi D, et al. 2008 French national guidelines for the treatment of venous thromboembolism in patients with cancer: Report from the working group. Crit Rev Oncol Hematol 2010;73(1):31-46. http://dx.doi.org/10.1016/j.critrevonc.2008.12.004

29. Mahé I, Chidiac J, Helfer H, Noble S. Factors influencing adherence to clinical guidelines in the management of cancer-associated thrombosis. J Thromb Haemost 2016;14(11):2107-2113. http:// dx.doi.org/10.1111/jth.13483

30. Miller CM, Ketlhapile M, Rybasack-Smith H, Rosen S. Why are antiretroviral treatment patients lost to follow-up? A qualitative study from South Africa. Trop Med Int Health 2010;15(s1):48-54. https: $/$ doi.org/10.1111\%2Fj.1365-3156.2010.02514.x

31. Boehringer Ingelheim Pharmaceuticals. Pradaxa (dabigatran etexilate mesylate): Highlights of Prescribing Information. Ridgefield, USA: Boehringer Ingelheim Pharmaceuticals, 2015. http:/ docs.boehringer-ingelheim.com/Prescribing\%20Information/PIs/Pradaxa/Pradaxa.pdf (accessed 25 February 2017).

32. Janssen Pharmaceuticals. Xarelto (rivaroxaban): Highlights of Prescribing Information. Leverkusen Janssen Pharmaceuticals. Xarelto (rivaroxaban): Highlights of Prescribing Information. Leverkusen,
Germany: Janssen Pharmaceuticals, 2016. https://www.xareltohcp.com/shared/product/xarelto Germany: Janssen Pharmaceuticals, 2016. https://ww
prescribing-information.pdf (accessed 25 February 2017).

33. Pfizer. Eliquis" (apixiban): Highlights of Prescribing Information. New York, USA: Pfizer, 2016. https:/ www.accessdata.fda.gov/drugsatfda_docs/label/2016/202155s012lbl.pdf (accessed 25 February 2017).

34. Joint United Nations Programme on HIV/AIDS. HIV and AIDS Estimates (2015). Geneva: UNAIDS, 2015. http://www.unaids.org/en/regionscountries/countries/southafrica (accessed 1 March 2017).

Accepted 21 April 2017. 